\title{
Research on the Model of Long Term Generation Planning in Power Market Reform
}

\author{
Bin Han1, Xiao-Lei Li', Jing-Hua Yan1, Hui Cui', Zhi-Qiang Luo² \\ ${ }^{1}$ China Electric Power Research Institute, Beijing, China \\ ${ }^{2}$ State Grid Corporation of China, Beijing, China \\ Email: 174318845@qq.com, Luo-zhiqiang@sgcc.com.cn
}

How to cite this paper: Han, B., Li, X.-L., Yan, J.-H., Cui, H. and Luo, Z.-Q. (2017) Research on the Model of Long Term Generation Planning in Power Market Reform. Energy and Power Engineering, 9, 525-534. https://doi.org/10.4236/epe.2017.94B058

Received: February 6, 2017

Accepted: March 30, 2017

Published: April 6, 2017

\begin{abstract}
With the deepening of China's power market, bilateral transactions will continue to grow in large scale. The release of bilateral transactions locked more regulatory resources of the power grid, will directly affect the operation mode of the unit and the implementation of planned electricity. In the paper, considering the large-scale bilateral trade effect on the peak regulation of power grid, energy saving and emission reduction, power system security and other factors, and then putting forward the method of long term generation planning and annual planning model to adapt to the safe operation of power grid in China. In the model, the target is minimizing the monthly load rate deviation and the annual electric quantity deviation rate, the latter includes the capacity factor. In addition, the constraints include the monthly quantity of electricity, adjustable utilization rate deviation, load rate, reserve and key sections, etc. Through an example to verify the correctness of the model, the planning and power transaction results can satisfy the peak regulation of load, energy saving and emission reduction and safety operation of the power grid requirements.
\end{abstract}

\section{Keywords}

Power Market, Generation Scheduling, Peak Regulation, Energy Saving and Emission Reduction, Power System Security

\section{Introduction}

In 2015, the State Council issued a number of opinions on further deepening the reform of power system [1].

In this paper, it will further promote the reform of the electric power trading system, improve the market trading mechanism, and guide the market players to carry out multi direct transactions, to encourage the establishment of long-term 
stable trading mechanism. The direction of future electric power system reform is clear, so it is expected that the future electricity bilateral transactions will increase in large scale.

As China's power market in the early stages of reform, the market mechanism is still not perfect, the processing mode of bilateral transaction is basically consistent with the planned electricity quantity, and it requires mandatory implementation. The liberalization of bilateral transactions has put forward a serious challenge to the current scheduling management [2].

1) The relation between stock and increment is more difficult. Bilateral transaction is closely related to the interests of the market players. When the load deviate, water regime changes, new energy power generation changes, and maintenance and other factors change, the planned power or power trading may not be fully implemented.

2) Operational control will be subject to greater constraints. With the expansion of trading volume, more power grid regulation resources are solidified. If the re-emergence of power grid maintenance, fault, bad weather and other factors, the adjustment of space will be further reduced, the difficulty and risk of grid operation increased significantly.

3) The new energy accommodation will be affected. At present, China's wind power, photovoltaic and other renewable energy is in a period of rapid growth. Power trading will lock a large number of high-quality resources and peak transmission resources, then, the system peak load capacity is restricted, the ability of power consumptive new energy will reduce.

At present, domestic and foreign research on the quantity decomposition of different periods in the year, month, day is more [3]-[11]. However, in view of China's bilateral trade liberalization, considering the system peak regulation, energy conservation, clean energy consumption, network security and other factors, guaranteeing planning and trading power coordinated implementation, the study of long-term power generation planning method and optimization model is relatively small.

This paper analyzes the medium and long term planning method after the release of bilateral trade, according to peak regulation, energy-saving emission reduction, the key section of constraints and other factors. First, peak regulation, energy saving and emission reduction, clean energy consumptive and other constraints is established, and then, target is to minimize the monthly load rate deviation and annual power deviation rate, which contains the capacity factor, finally, the model of the annual plan is established.

In this paper, the mixed integer programming method is used to solve the model. Solving software is CPLEX, which is practical and efficient.

\section{Analysis of the Factors of Preparation Method}

\subsection{Peak Regulation of Power Grid}

The execution of the power trade and the planning quantity should consider peak regulation of power grid. The main basis is: "power law", "renewable ener- 
gy law", "power grid dispatching management regulations", "power plant auxiliary service management Interim Measures" and so on. It must give priority to the clean energy consumptive, ensure all power enterprises be fair to undertake peak frequency and reserve, to meet the needs of power peaking.

Consider method:

1) Power generation companies involved in direct trading, will certainly achieve more power generation, higher utilization hours, however, it must be controlled in a certain extent, to avoid generating stop preparation, peak regulation service unequal and unfair.

2) For inter provincial direct power purchase transactions, the conventional transaction curve peak valley ratio of the thermal power unit should be higher than the peak valley ratio of the receiving end power grid.

3) Due to the difference of the maintenance period of each generating unit, the power generation unit with less direct access to the maintenance work should be involved in the direct transaction, so that the utilization rate of the power generation unit can be adjusted to a balance.

4) For the direct purchase of electricity transactions in the province, the gap between the adjustable utilization rate of the power generation unit and adjustable utilization rate of the whole power grid should not be too large.

\subsection{Energy Saving and Emission Reduction}

In the medium and long term power generation plan, the clean energy generation space should be considered. The main basis is the "renewable energy law" and "wind power priority scheduling work norms". It clearly requires that the amount of clean energy in the medium and long term plan.

Consider method:

1) In the power generation plan, the power generation utilization hours of the power grid should be consistent with the unit capacity level. High parameter, large capacity thermal power generation should be priority.

2) Medium and long term forecast of clean energy power generation should be taken into account.

\subsection{Network Constraints}

In the medium and long term power generation plan, the blocking problem of the transmission section should be considered, to ensure the implementation of bilateral trade and planned quantity.

Consider method:

1) Because of the difference in annual and monthly in accuracy, the method of considering the network constraints in the long-term power constraint network plan is mainly based on the typical operation mode of power grid to calculate the sensitivity, and then calculate the limit value of the key section.

2) The typical operation mode will be affected by the variation of unit operation mode in actual operation, which caused by Equipment inspection and load mutation, so the constraint of transmission section cannot be accurate, it must 
be kept in a certain margin.

\section{Model Construction}

\subsection{Objective Function}

The model constructed in this paper is to solve the trading and planning quantity of each month.

The implementation of the plan quantity is based on the principle of openness, fairness and impartiality. If in accordance with the traditional method of scheduling the average distribution, in some months, the unit's planned electricity may not be guaranteed to perform, due to wind power and other clean energy generation change or unit maintenance and other reasons. Therefore, the method is based on the medium and long term load forecasting data, according to the proportion of load distribution plan.

Distribution of bilateral transactions meet the contract provisions of the implementation of the monthly rate. If there is no such provision in the contract, the processing method is to allocate the quantity of electricity in accordance with the load ratio, similar to planning quantity.

Considering the peak load regulation and energy saving and emission reduction, network with multiple constraints, trading or plan quantity of unit may not be able to ensure full implementation. Therefore, the quantity of the medium and long term trading plan should be considered as a soft constraint in the model. The consideration method is to consider the minimum of the power deviation rate of the unit in the model.

According to the requirements of the above arrangement unit generating capacity to be consistent with the unit capacity, this paper increases the capacity factor of the unit in the target of minimum power deviation rate.

So, the model objectives are as follows:

$$
\min F=\sum_{i=0}^{N} \sum_{j=0}^{T}\left|\frac{Q_{i, j}}{Q_{i}}-\gamma_{i, j}\right|+\sum_{i=0}^{N} \frac{\mu_{i}\left|Q_{i}-\sum_{j=0}^{T} Q_{i, j}\right|}{Q_{i}}
$$

Among it,

$$
\gamma_{i, j}=\left\{\begin{array}{l}
\frac{P_{j}}{\sum_{j=0}^{T} P_{j}} \\
\gamma_{j}
\end{array}\right.
$$

In the formula, $\mathrm{N}$ represents the total number of units that have bilateral trade or planning quantity, $\mathrm{T}$ represents the number of time periods in the study period, $Q_{i}$ represents the total quantity of the transaction or the planning for the first $i$ unit, $Q_{i, j}$ represents the $j$ monthly quantity of the transaction or the planning for the first $i$ unit, $\mu_{i}$ represents the unit capacity factor, which is proportional to the unit capacity.

$\gamma_{i, j}$ represents the $j$ monthly quantity distribution rate of the $i$ unit, and $P_{j}$ indicates the load of the system for $J$ months. When the transaction contract provides for the month of the electricity distribution rate of $\gamma_{j}, \gamma_{i, j}$ is equal to 
$\gamma_{j}$, or equal to the system load distribution rate.

\subsection{Constraint Condition}

1) Upper and lower bound constraints on trading or planning quantity of unit:

$$
Q_{i, j}^{\min }<Q_{i, j}<Q_{i, j}^{\max }
$$

In the formula, $Q_{i, j}^{\min }$ and $Q_{i, j}^{\max }$ distribution indicates the minimum and maximum values of $j$ monthly trading or planning quantity for the $i$ unit.

Among it, the maximum power generation capacity of the unit is the maximum power generation that does not contain the unit maintenance capacity. The minimum electricity quantity of a unit is the minimum execution quantity or zero.

2) Upper and lower bound constraints on adjustable utilization deviation of unit's trading or planning quantity:

$$
\Delta_{i}^{\min }<\left|\frac{\sum_{j=0}^{T} Q_{i, j}}{Q_{i}^{\max }}-\frac{\sum_{i=0}^{N} \sum_{j=0}^{T} Q_{i, j}}{\sum_{i=0}^{N} Q_{i}^{\max }}\right|<\Delta_{i}^{\max }
$$

In the formula, $\Delta_{i}^{\min }$ and $\Delta_{i}^{\max }$ distribution indicates the minimum and maximum values of adjustable utilization deviation.

The constraint indicates that the gap between the adjustable utilization rate of the unit and the adjustable utilization rate of the system can be adjusted in the control range.

3) Upper and lower bound constraints on load rate of unit:

$$
\delta_{i}^{\min }<\frac{\sum_{j=0}^{T} Q_{i, j}}{Q_{i}^{\max }}<\delta_{i}^{\max }
$$

In the formula, $\delta_{i}^{\min }$ and $\delta_{i}^{\max }$ distribution indicates the minimum and maximum values of unit's load rate.

The constraint indicates that unit's load rate can be adjusted in the control range.

4) Reserve constraint:

$$
\varepsilon_{i}^{\min }<P_{j}-\sum_{i=0}^{N} Q_{i, j}<\varepsilon_{i}^{\max }
$$

In the formula, $\varepsilon_{i}^{\min }$ and $\varepsilon_{i}^{\max }$ indicates the minimum and maximum values of unit's reserve.

The constraint indicates that All quantity of units to meet the reserve constraint, in order to ensure the clean energy consumptive space.

5) The key section power flow constraint:

$$
C_{k}^{\min }<\alpha \mathrm{C}_{k}\left(Q_{i, j}\right)<C_{k}^{\max }
$$

In the formula, $C_{k}^{\min }$ and $C_{k}^{\max }$ indicates the minimum and maximum values of the $k$ key section. $\mathrm{C}_{k}\left(Q_{i, j}\right)$ indicates the power function of the power of the $k$ transmission section on the $j$ month of the $i$ unit. It is mainly based on the typical operation mode of power grid to calculate the sensitivity, and then calculate the limit value of the key section. 
$\alpha$ indicates power adjustment factor of transmission section. It is mainly based on the typical operation mode of power grid. Due to the variation of the maintenance plan, load and new energy, the accuracy is different, so the constraint of the transmission section must have a certain margin. In this paper, $\alpha$ is set $90 \%$.

\section{Analysis of Example}

In the example, the goal is to calculate units trading or planning quantity for each month in a year. In this paper, the load and the reserve data in a certain area are shown in the following Table 1.

As seen from the table, this paper sets up the reserve to take into account the situation, in which the high power of hydropower in summer and the high power of wind-power in winter.

This paper selects 7 different capacity units from the area, the unit data is shown in the following Table 2. The unit maintenance plan is not considered in the calculation example. In addition, the unit 2 is in an important section.

Table 1. Load and reserve data.

\begin{tabular}{ccc}
\hline month & Load $(\mathrm{MWH})$ & Reserve $(\mathrm{MWH})$ \\
\hline 1 & $8,104,914$ & $3,241,966$ \\
2 & $6,599,596$ & $1,979,879$ \\
3 & $7,506,299$ & $2,251,890$ \\
4 & $7,855,502$ & $2,356,651$ \\
5 & $8,418,932$ & $2,525,680$ \\
6 & $7,475,207$ & $2,990,083$ \\
7 & $7,817,300$ & $3,126,920$ \\
8 & $5,595,628$ & $2,238,251$ \\
9 & $6,433,157$ & $1,929,947$ \\
10 & $7,227,234$ & $2,168,170$ \\
11 & $7,938,806$ & $3,175,522$ \\
12 & $6,240,151$ & $2,496,060$ \\
\hline
\end{tabular}

Table 2. Unit data.

\begin{tabular}{ccccc}
\hline unit & capacity (MW) & quantity 1 (MWH) & quantity 2 (MWH) & quantity 3 (MWH) \\
\hline unit 1 & 1000 & $2,628,000$ & $3,504,000$ & $4,380,000$ \\
unit 2 & 800 & $2,102,400$ & $2,803,200$ & $3,504,000$ \\
unit 3 & 600 & $1,576,800$ & $2,102,400$ & $2,628,000$ \\
unit 4 & 500 & $1,314,000$ & $1,752,000$ & $2,190,000$ \\
unit 5 & 300 & 788,400 & $1,051,200$ & $1,314,000$ \\
unit 6 & 200 & 525,600 & 700,800 & 876,000 \\
unit 7 & 135 & 354,780 & 473,040 & 591,300 \\
\hline
\end{tabular}


When the quantity of the trading or planning is smaller, the results of the case are shown in Figure 1.

The quantity result can ensure the completion of trading or planning quantity, meet all kinds of constraints in the model, and the completion rate of the power in each month is basically consistent with the load rate of each month.

When the quantity of the trading or planning is bigger, the results of the case are shown in Figure 2.

The quantity results can ensure the completion of the transaction plan to meet all kinds of constraints in the model, but the power consumption in the completion rate of each month and the monthly load rate of the basic gap is relatively large. The reason is that the weight of the load rate deviation is small. The research focus of this paper is to ensure the completion rate of the trading or planning under the premise of satisfying all kinds of constraints, the target of

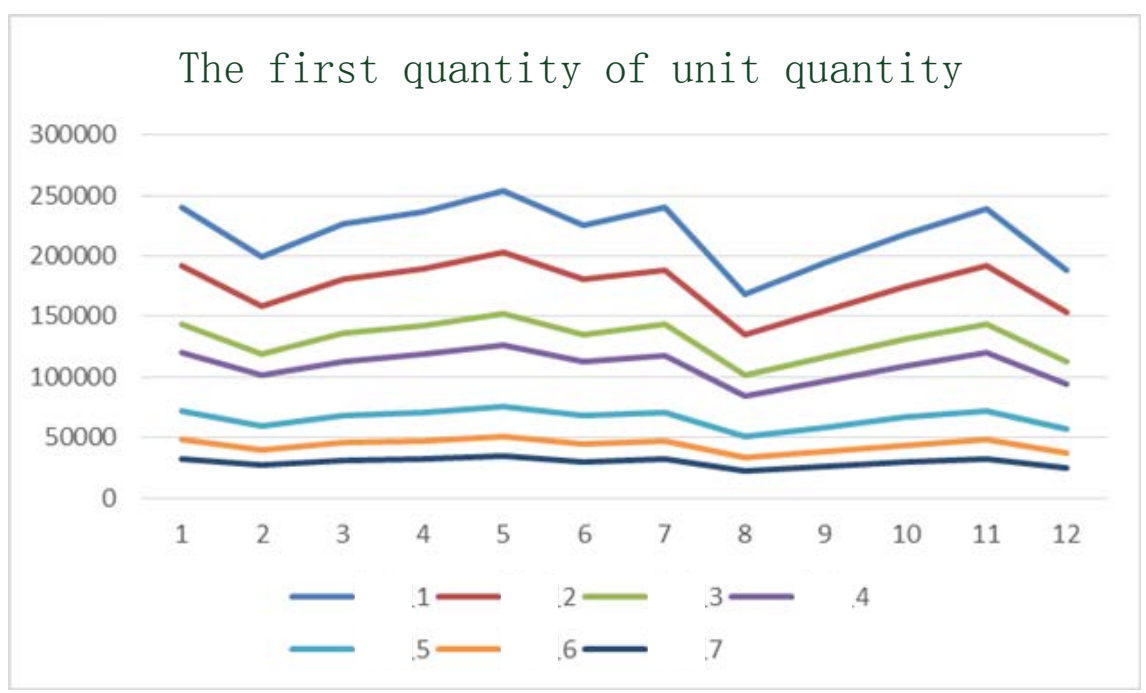

Figure 1. The first result of monthly electric quantity.

\section{The second quantity of unit quantity}

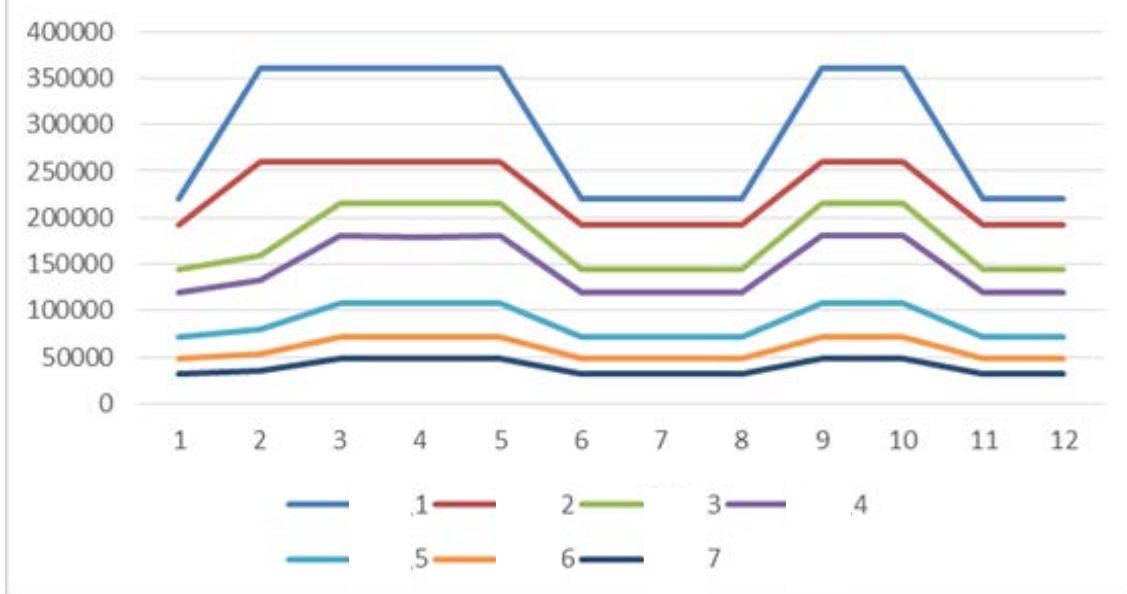

Figure 2. The second result of monthly electric quantity. 
minimum load deviation is in the secondary position. If the load rate is guaranteed to be guaranteed, it can be achieved by increasing the weight factor in the target.

In addition, the Figure 2 shows that the quantity value from February to May, and in September and October reach the upper limit of the section, while the remaining months are subject to other constraints, eventually leading to the final annual electricity cannot be completed.

When the quantity of the trading or planning is greater, the results of the case are shown in Figure 3.

Unit electric quantity completed as shown in Table 3.

The Table 3 shows that when the quantity is too large, the system requirements of all kinds of constraints cannot be met, it is required to cut quantity of trading or planning. The principle of reduction is basically consistent with the capacity of the unit, and the calculation of the amount of the units of each month to reach the upper limit of all kinds of constraints.

The unit 2 is subjected to the constraint of the section, which leads to the low load rate of the unit.

\section{The third quantity of unit quantity}

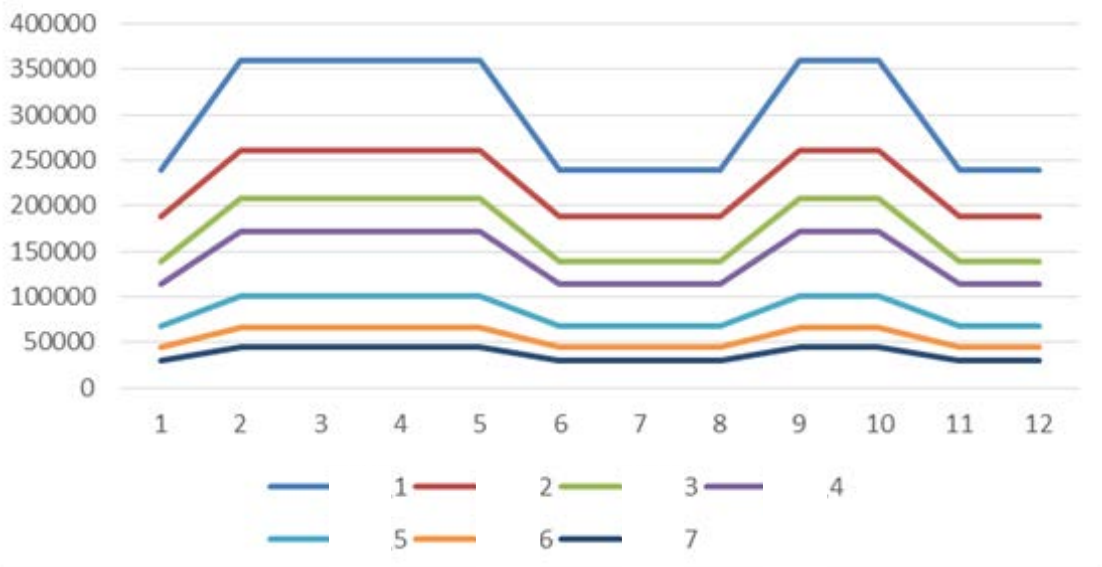

Figure 3. The Third result of monthly electric quantity.

Table 3. The third result of quantity completion.

\begin{tabular}{ccc}
\hline & Completion rate & Completed quantity $(\mathrm{MWH})$ \\
\hline unit 1 & 0.821918 & $3,600,000$ \\
unit 2 & 0.767397 & $2,688,960$ \\
unit 3 & 0.789041 & $2,073,600$ \\
unit 4 & 0.780822 & $1,710,000$ \\
unit 5 & 0.764384 & $1,004,400$ \\
unit 6 & 0.756164 & 662,400 \\
unit 7 & 0.750822 & 443,961 \\
\hline
\end{tabular}




\section{Conclusions}

Based on the current laws and regulations of our country, this paper studied the method of the medium and long term power generation plan adapting to the development of China's electric power market, a medium and long term power generation planning model is proposed to meet the liberalization of bilateral trade. The model takes into account the peak and energy-saving emission reduction and the key section constraints, in which the load rate deviation and the electric quantity deviation are taken as the target.

By example, the model can ensure that the establishment of bilateral trade and planning power can fully consider the new energy generation space, energy conservation and emission reduction, power grid safe operation and other factors.

\section{Acknowledgements}

The paper is supported by State Grid Corporation of China. The research funding comes from the related science and technology project, which is "the key technology research of the power generation scheduling in the large-scale bilateral transaction environment".

\section{References}

[1] (2015)A Number of Opinions on Further Deepening the Reform of Power System. Central Committee and State Council.

[2] Leng, Y., Chen, Z., Wang, Q.H., et al. (2015) Analysis of the Impact of Direct Power Purchase by Large Consumers in CSG Area and Relative Suggestions. Southern Power System Technology, 9. (in Chinese).

[3] Wang, Y.L., Ding, P., Liu, X., et al. (2002) Coordination of the Dispatching Relationship between the Contract Volume and Competitive Bidding Volume in Daily Generation Scheduling. Automation of Electric Power Systems,26, 45-48.(in Chinese)

[4] Jiang, D.R., Liu, X.J. and Li, Q.Z. (2002) Economically Distributing Strateges for Daily Generation Scheduling in a Power System under power Market Environment. Proceedings of the CSEE, 24, 90-94. (in Chinese)

[5] Lv, Q., Li, W.D. and Wu, Y.G. (2005) A Novel Mutiperiod Transaction Algorithm for Day-Ahead Electricity Market. Power System Technology, 29, 33-37. (in Chinese)

[6] Chu, Z. and Yu, J.L. (2006) Model and Method for Daily Dispatch Scheduling in Primary Power Markets. Automation of Electric Power Systems, 30, 43-47. (in Chinese)

[7] Yu, Z., Sparrow, F.T. and Nderitu, D. (1998) Long-term Hydrothermal Scheduling Using Composite Thermal and Composite Hydro Representations. IEEE Proceedings of Generation Transmission Distribution, 145,210-216.

https://doi.org/10.1049/ip-gtd:19981794

[8] Ikuo, K., Hiroshi, A., Kenji, O., et al. (2002) Long-term Power Trade Model in Electricity Market Based on Game Theory. Proceedings of the IEEE Power Engineering Society Transmission and Distribution Conference, Yokahama, Japan.

[9] Fu, Y., Shahidehpour, M. and Li, Z.Y. (2005) Long-term Security -Constrainedunit Commitment Hybrid Dantzig-Wolfe Decomposition and Subgradient Approach. 
IEEE Trans on Power Systems, 20, 2093-2106.

https://doi.org/10.1109/TPWRS.2005.857286

[10] Wang, Y., Yu, J.L. and Liu, Z. (2006) Decomposition of Yearly Bided Volume Based on Roll-Uniformization of Monthly Competitive Bided Spaces. Automation of Electric Power Systems, 30, 24-27. (in Chinese)

[11] Tang, W., Wang, Y., Yu, F., et al. (2009) An Integrative Cost Weighted Method for Drawing Up Electric Energy Monthly Transaction Schedule of Directly Dispatched Thermal Power Generation Units. Power System Technology, 33, 167-173(in Chinese).

Submit or recommend next manuscript to SCIRP and we will provide best service for you:

Accepting pre-submission inquiries through Email, Facebook, LinkedIn, Twitter, etc. A wide selection of journals (inclusive of 9 subjects, more than 200 journals) Providing 24-hour high-quality service User-friendly online submission system Fair and swift peer-review system Efficient typesetting and proofreading procedure Display of the result of downloads and visits, as well as the number of cited articles Maximum dissemination of your research work

Submit your manuscript at: http://papersubmission.scirp.org/ Or contact epe@scirp.org 